\title{
Prognostic Factors for Evolution of Non Alcoholic Fatty Liver Disease Patients Utilizing Poisson Regression and Continuous Time Markov Chains
}

\author{
Iman M. Attia * \\ Imanattiathesis1972@gmail.com,imanattia1972@gmail.com \\ *Department of Mathematical Statistics, Faculty of Graduate Studies for Statistical Research, Cairo University, Egypt
}

\begin{abstract}
In the present paper, the deleterious effects of obesity, type 2diabetes and insulin resistance, systolic and diastolic hypertension on the rate of progression of fibrosis in nonalcoholic fatty liver disease (NAFLD) patients are illustrated using a new approach utilizing the Poisson regression to model the transition rate matrix. The observed counts in the transition counts matrix are used as response variables and the covariates are the risk factors for fatty liver. Then the estimated counts from running the Poisson regression are used to estimate the transition rates using the continuous time Markov chains (CTMC) followed by exponentiation of the estimated rate matrix to obtain the transition probability matrix at specific time points. Using a hypothetical data of $\mathbf{1 5 0}$ participants followed up every year for a total of 28 years recording their demographic characteristics and their timeline of follow up are demonstrated. The findings revealed that insulin resistance expressed by MOMA-IR 2 has the most deleterious effects among other factors for increasing the rate of forward progression of patients from state 1 to state 2 as well as from state 2 to state 3 and from state 3 to state 4 . The higher the level of HOMA-IR is, the more rapid the rate of progression is.

Key words: Continuous time Markov chains, Life expectancy, Maximum Likelihood estimation, Mean Sojourn Time, NonAlcoholic Fatty Liver Disease, Panel Data.
\end{abstract}

\section{INTRODUCTION}

Continuous time Markov chains (CTMC) are valuable and of great potentiality mathematical and statistical tools to be used for evaluation of disease progression over time. CTMCs are a subtype of multistate models to be utilized to study this progression in NAFLD patients, with its characteristic phenotypes NAFLD and NASH, hand in hand with the presence of associated fibrosis and its stages. The prevalence of NAFLD is quickly growing worldwide, and matches the epidemics of obesity and type 2 diabetes. Metabolic syndrome is a well-known risk factor which requires the presence of abdominal obesity distinguished by waist circumference $>94$ $\mathrm{cm}$ for males and $>80 \mathrm{~cm}$ for females in eastern countries while it is $>120 \mathrm{~cm}$ for males and $>88 \mathrm{~cm}$ for females in the western countries, plus 2 or more of the following: blood glucose $\geq 100$ $\mathrm{mg} / \mathrm{dL}$ or drug treating diabetes, arterial blood pressure $\geq$ $130 / 85 \mathrm{mmgh}$ or drug treating hypertension, triglyceride levels $\geq 150 \mathrm{mg} / \mathrm{dL}$ or drug treating increased levels in blood or high density lipoprotein (HDL) levels $<40 \mathrm{mg} / \mathrm{dL}$ for males and $<50$ $\mathrm{mg} / \mathrm{dL}$ for females or drug treating this condition.

NAFLD can be modeled using the simplest form for health, disease, and death model, with one state for susceptible individuals with risk factors, such as: type 2 diabetes, dyslipidemia and hypertension, the other state is the NAFLD phenotypes, and two competing states for death: one for liverrelated mortality as a complication of NAFLD, and the other death state is death causes unrelated to liver disease [1]. This is shown in figure 1:

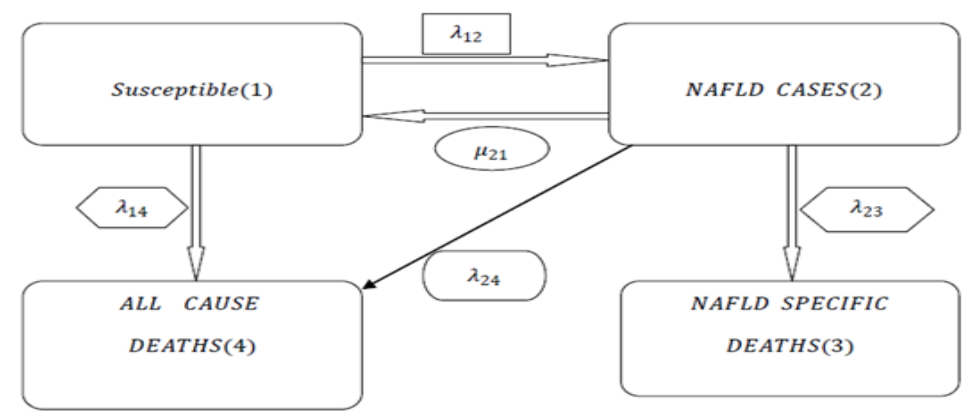

Figure 1: General Model Structure 
In addition, NAFLD is modeled in more elaborative expanded form, which includes nine states: the first eight states are the states of disease progression as time elapses, while the ninth state is the death state [1], as illustrated in figure 2:

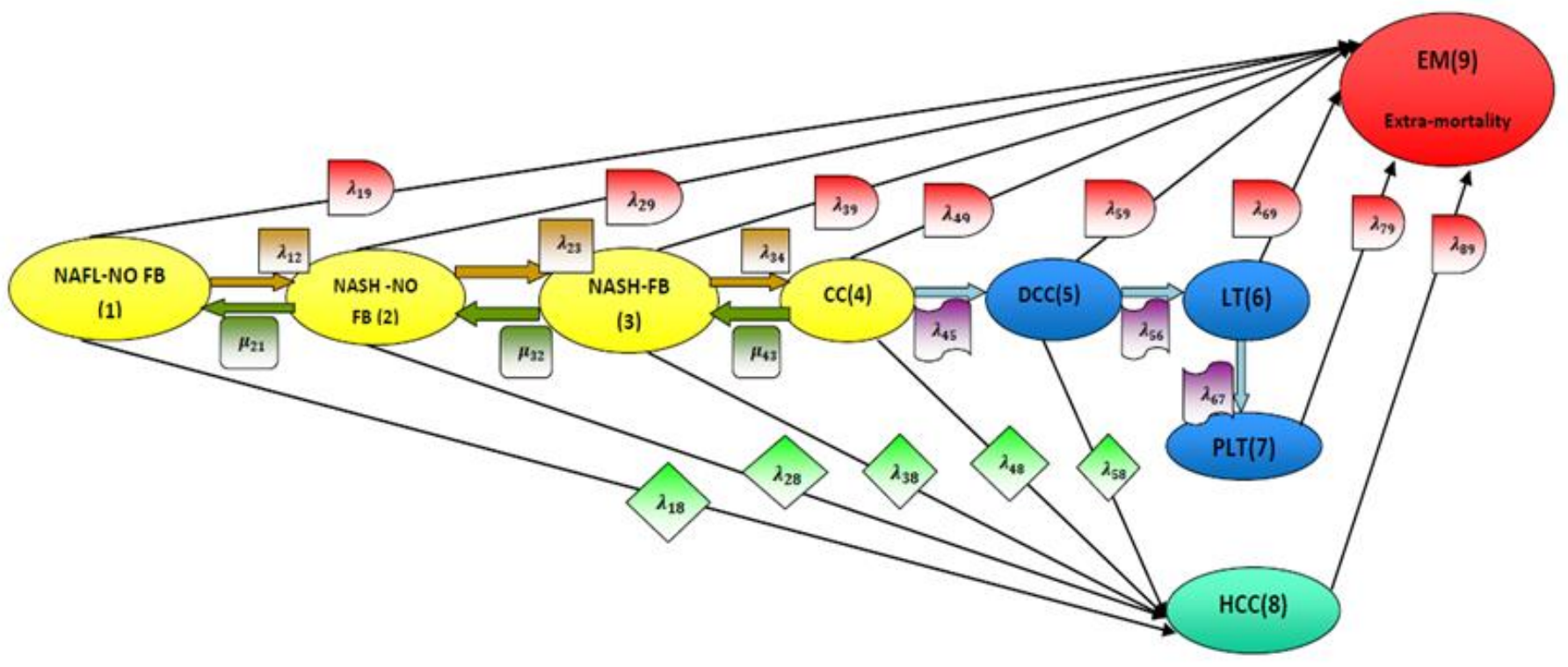

Figure 2: disease model structure:

NAFLD-NO FB: nonalcoholic fatty liver disease with no fibrosis (stage 1). NASH-NO FB: nonalcoholic steatohepatitis with no fibrosis (stage 2). NASH-FB: nonalcoholic steatohepatitis with fibrosis (stage 3). CC: compensated cirrhosis (stage 4). DCC: decompensated cirrhosis (stage 5). LT: liver transplant (stage 6). PLT: post liver transplant (stage 7). HCC: hepato-cellular carcinoma ( stage 8). EM: extramortality (stage 9).

Moreover, a subset of the states that explicitly illustrates the phases of fibrosis process, which develops early in disease evolution cycle if the risk factors are not treated or eliminated, is modeled with CTMC to demonstrate: how covariates incorporated in a log-linear model can relate these predictors to transition rates among states, as illustrated in figure 3 [2],[3].
The presence of fibrosis is considered an ominous predictor for disease progression. This subset is a subset of states from the expanded model especially early phases or stages where reversibility of conditions in each stage can be achieved if properly treated and controlled so as to prevent reaching the irreversible damaged state which is liver cirrhosis or F4.

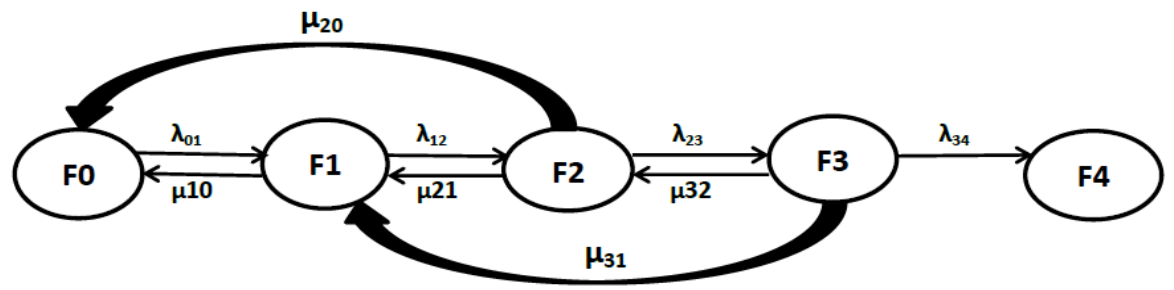

Figure 3: NAFLD with the evolving fibrosis stages.

$F O=$ no fibrosis (stage 0) whether hepatic steatosis is present or not. NASH-FB-1: nonalcoholic steatohepatitis with mild fibrosis (stage 1). NASH -FB-2: NASH with moderate fibrosis (stage 2). NASH -FB-3: NASH with advanced or severe fibrosis (stage 3). CC: compensated cirrhosis (stage 4) which is the more severe or advanced form of fibrosis.

Singh et al. 2015 conducted a meta-analysis to evaluate the rate of fibrosis progression and thus searched multiple databases through a thoroughly systematic manner associated with author contact and found 11 cohort studies on NAFLD adult patients having at least one year apart paired liver biopsy specimens, from which they calculated a pooled-weighted annual fibrosis progression rate (number of stages changed between the 2 biopsy samples) with $95 \%$ confidence interval (CIs), and characterized the clinical risk factors accompanying this progression. They identified 411 patients with biopsy-proven
NAFLD (150 with NAFL and 261 with NASH) included in those studies. Initially, the distribution of fibrosis for stages $0,1,2,3$ and 4 was $35.8 \%, 32.5 \%, 16.7 \%, 9.3 \%$ and $5.7 \%$ respectively, and over 2145.5 person-years of follow-up evaluation, $33.6 \%$ had fibrosis progression, $43.1 \%$ had stable fibrosis, and $22.3 \%$ had an improvement in fibrosis stage. The annual fibrosis progression rate in patients with NAFL who had stage 0 fibrosis at baseline was .07 stages (95\% CI, 0.02-0.11 stages), compared with 0.14 stages in patients with $\mathrm{NASH}$ (95\% CI, 0.07-0.21 stages). These findings correspond to 1 
stage of progression over 14.3 years for patients with NAFL (95\% CI, 9.1-50.0 y) and 7.1 years for patients with NASH (95\% CI, 4.8-14.3 y).

Kalbfleisch and Lawless [4] related the instantaneous rate of transitions from state $i$ to state $j$ to covariates, by regression modeling of the $\mathrm{Q}$ transition rate matrix using log-linear model for the Markov rates.

In the present study, Poisson regression is used to model the rates among states. The counts of each transition can be modeled as a function of some explanatory variables reflecting the characteristics of the patients. This can be accomplished by using Poisson regression model or log-linear model. The Poisson regression model specifies that each response $y_{i}$ is drawn from a Poisson population with parameter $\lambda_{i}$, which is related to the regressors or the covariates. The primary equation of the model is

$$
P\left(Y=y_{i} \mid x_{i}\right)=\frac{e^{-\lambda_{i}} \lambda_{i}^{y_{i}}}{y_{i} !}
$$

The most common formulation for the $\lambda_{i}$ is the log-linear model:

$$
\ln \lambda_{i}=x_{i}^{\prime} B
$$

And the expected number of events per period is given by:

$$
E\left[y_{i} \mid x_{i}\right]=\operatorname{var}\left[y_{i} \mid x_{i}\right]=\lambda_{i}=e^{x_{i}^{\prime} B}
$$

The observed counts in the transition counts matrix is used as response variables and the covariates are the risk factors for fatty liver. Then the estimated counts obtained from the Poisson regression model are used to estimate the rates using the CTMC, as the initially observed transition rates approximately equal the estimated transition rates among states, as illustrated by the author in previous 2 papers, followed by exponentiation of the estimated rate matrix. To expound this procedure a hypothetical example is used, and it is in the form of a study conducted on 150 participants over 28 years to follow the progression of the NAFLD from F0 to F4.

The paper is divided into 3 sections. In section 1, illustration of the study design is clarified. In section 2, the results and discussion of running the Poisson regression model is elucidated. In section 3 , conclusion of the running this model is expounded. Supplementary materials are complementary to this paper as some information are strictly presented in these materials and not in this main paper, such materials are table1,6,8,23, and figures from figure 13 to figure 21 .

\section{Study Design}

One hundred fifty participants were followed up every year for 28 years, and at each visit the characteristics of the participants were recorded like $\operatorname{sex}(0=$ female, $1=$ male $)$, age, BMI, LDL-chol, HOMA2_IR, systolic blood pressure as well as the diastolic pressure as shown in the table (1) (see supplementary materails). For each participant the recorded value in the table is the mean of the follow up measurements. Fitting the Poisson regression and the estimated counts for each transition were calculated using Stata 14. A summary statistics for the patients' characteristics is shown in table (2). The participants were categorized according to these demographic characteristics as shown in table (3), while in table (4) summary of the categorical groups according to the participants' characteristics like: age category BMI category, LDL-chol category, systolic and diastolic blood pressure category. There are high correlations between the continuous predictor variables as shown in table (5). In table (6) (see supplementary materials) the transition counts accomplished by each participant in these 28 years are illustrated. Summary of transition counts among the states in these 28 years is clarified in table (7). The timeline for each participant is shown in table (8) ( see supplementary materials) with first column is $\mathrm{t}=0$ and the last column is $\mathrm{t}=28$ and in each of these column(year) the state of the patient was recorded. The observed transition counts are illustrated in table (9).

The distribution of the transition counts among the states is Poisson as illustrated in the following successive figures using Statgraphics-19 software. For transition from 0 to 1 , see figure (4). For transition from 1 to 2, see figure (5). For transition from 2 to 3, see figure (6). For transition from 3 to 4 , see figure (7). For transition from 1 to 0 , see figure (8). For transition from 2 to 1 , see figure (9). For transition from 3 to 2 , see figure (10). For transition from 2 to 0 , see figure (11). For transition

\begin{tabular}{|c|c|c|c|c|c|}
\hline Variable & Observations & mean & Std. Dev. & Min & Max \\
\hline \multirow{3}{*}{$\begin{array}{c}\text { Gender: } \\
\text { Female }=0 \\
\text { Male }=1\end{array}$} & 150 & & & & \\
\hline & $69(0.46)$ & & & & \\
\hline & $81(0.54)$ & & & & \\
\hline Age & 150 & 40.2 & 4.93 & 27 & 53 \\
\hline LDL-chol & 150 & 94.81 & 15.41 & 59.89 & 133.1 \\
\hline HOMA2-IR & 150 & 2.28 & .71 & .49 & 4.36 \\
\hline BMI & 150 & 28.28 & 2.991 & 20.3 & 35.16 \\
\hline Sys.Bl.Pr. & 150 & 149.73 & 10.434 & 123.4 & 175.75 \\
\hline Dias.Bl.Pr. & 150 & 94.25 & 11.39 & 70 & 124 \\
\hline
\end{tabular}
from 3 to 1 , see figure (12).

Table (2): statistical summary of the patients' characteristics 
Table (3): table summarizing the categorical groups of patients according to the previous characteristics

\begin{tabular}{|c|c|c|c|}
\hline Variable & Group1 (desirable) & Group2 (borderline) & Group3 (high) \\
\hline Age & Age $\leq 35$ & $35<$ age $\leq 45$ & Age $>45$ \\
\hline LDL-chol & LDL $\leq 70$ & $70<$ LDL $<100$ & LDL $\geq 100$ \\
\hline HOMA2-IR & HOMA $<1.22$ & $1.22 \leq \mathrm{HOMA}<2.7$ & HOMA $\geq 2.7$ \\
\hline BMI & BMI $\leq 25$ & $25<$ BMI $<30$ & BMI $\geq 30$ \\
\hline $\begin{array}{c}\text { Systolic blood pressure } \\
\text { Diastolic blood } \\
\text { pressure }\end{array}$ & Sys.Pr. $\leq 130$ & $130<$ Sys.Pr. $<160$ & Sys.Pr. $\geq 160$ \\
\hline Dias. Pr. $\leq 85$ & $85<$ Dias. Pr. $<100$ & Dias. Pr. $\geq 100$ \\
\hline
\end{tabular}

Table (4): summary of categorical groups of the patients' characteristics regarding age category, BMI category, LDL-chol category, systolic and diastolic blood pressure category:

\begin{tabular}{|c|c|c|c|c|c|c|c|c|c|}
\hline \multirow{2}{*}{ category } & \multicolumn{3}{|c|}{ Age } & \multicolumn{3}{|c|}{ BMI } & \multicolumn{3}{|c|}{ LDL-chol } \\
\hline & Frequency & Percent & Cum. & Frequency & Percent & Cum. & Frequency & Percent & Cum. \\
\hline 1 & 22 & 14.67 & 14.67 & 22 & 14.67 & 14.67 & 5 & 3.33 & 3.33 \\
\hline 2 & 109 & 72.67 & 87.33 & 83 & 55.33 & 70 & 93 & 62.00 & 65.33 \\
\hline 3 & 19 & 12.67 & 100 & 45 & 30 & 100 & 52 & 34.67 & 100 \\
\hline total & 150 & 100 & & 150 & 100 & & 150 & 100 & \\
\hline \multirow{2}{*}{ category } & \multicolumn{3}{|c|}{ HOMA2-IR } & \multicolumn{3}{|c|}{ Systolic Blood Pressure } & \multicolumn{3}{|c|}{ Systolic Blood Pressure } \\
\hline & Frequency & Percent & Cum. & Frequency & Percent & Cum. & Frequency & Percent & Cum. \\
\hline 1 & 10 & 6.67 & 6.67 & 4 & 2.67 & 2.67 & 33 & 22 & 22 \\
\hline 2 & 93 & 62.00 & 68.67 & 123 & 82.00 & 84.67 & 69 & 46 & 68 \\
\hline 3 & 47 & 31.33 & 100 & 23 & 15.33 & 100 & 48 & 32 & 100 \\
\hline total & 100 & 100 & & 150 & 100 & & 150 & 100 & \\
\hline
\end{tabular}

Table (5): correlation between continuous predictor variables

\begin{tabular}{|c|c|c|c|c|c|c|}
\hline & age & LDL-chol & HOMA2-IR & BMI & Sys. Bl.Pr. & Dias. Bl.Pr. \\
\hline Age & 1 & & & & & \\
\hline LDL-chol & .9919 & 1 & & & & \\
\hline HOMA2-IR & .9941 & .9947 & 1 & & & \\
\hline BMI & .9938 & .9948 & .996 & 1 & & \\
\hline Sys. Bl.Pr. & .9958 & .9953 & .9958 & .9962 & 1 & .9949 \\
\hline Dias. Bl.Pr. & .9915 & .9951 & .9962 & .9945 & .996 \\
\hline
\end{tabular}

Table (7): summary transition counts between the states

\begin{tabular}{|c|c|c|c|c|c|c|c|c|c|}
\hline Counts & $\begin{array}{c}\text { Transition } \\
0 \rightarrow 1\end{array}$ & $\begin{array}{c}\text { Transition } \\
1 \rightarrow 2\end{array}$ & $\begin{array}{c}\text { Transition } \\
2 \rightarrow 3\end{array}$ & $\begin{array}{c}\text { Transition } \\
3 \rightarrow 4\end{array}$ & $\begin{array}{c}\text { Transition } \\
1 \rightarrow 0\end{array}$ & $\underset{2 \rightarrow 1}{\text { Transition }}$ & $\begin{array}{c}\text { Transition } \\
3 \rightarrow 2\end{array}$ & $\begin{array}{c}\text { Transition } \\
2 \rightarrow 0\end{array}$ & $\begin{array}{c}\text { Transition } \\
3 \rightarrow 1\end{array}$ \\
\hline $\mathbf{0}$ & 63 & 96 & 121 & 128 & 121 & 127 & 130 & 138 & 139 \\
\hline 1 & 58 & 43 & 23 & 22 & 24 & 17 & 17 & 11 & 9 \\
\hline 2 & 25 & 9 & 4 & & 3 & 5 & 3 & 1 & 2 \\
\hline 3 & 4 & 2 & 2 & & 2 & 1 & & & \\
\hline
\end{tabular}

Table (9): Observed transitions counts of the patients over the 28 years

\begin{tabular}{|c|c|c|c|c|c|c|}
\hline & State 0 & State1 & State2 & State3 & State4 & 0 \\
\hline State0 & 1909 & 120 & 15 & 6 & 2050 \\
\hline State1 & 36 & 1116 & 703 & 28 & 0 \\
\hline State2 & 13 & 30 & 23 & 57 & 0 \\
\hline State3 & 11 & 14 & 0 & 0 & 22 & 0 \\
\hline State4 & 0 & 0 & & & 0 \\
\hline
\end{tabular}

Initial observed rates are:

$$
\begin{aligned}
& \lambda_{01}=\frac{120}{2050}=.059, \lambda_{12}=\frac{67}{1247}=.0537, \lambda_{23}=\frac{37}{783}=.047, \lambda_{34}=\frac{22}{120}=.183 \\
& \mu_{10}=\frac{36}{1247}=.0288, \mu_{21}=\frac{30}{783}=.0383, \mu_{32}=\frac{23}{120}=.191, \mu_{20}=\frac{13}{783}=.016, \mu_{31}=\frac{14}{120}=.116
\end{aligned}
$$


Using CTMC, the estimated rates approximately equal the initially observed rates, as illustrated by the author Iman Attia in previous 2 papers utilizing the simplest small model and the expanded model, where no covariates were included in the analysis.[5]

The distribution of the transition counts is Poisson as illustrated in the following figures using the Statgraphics-19 software.

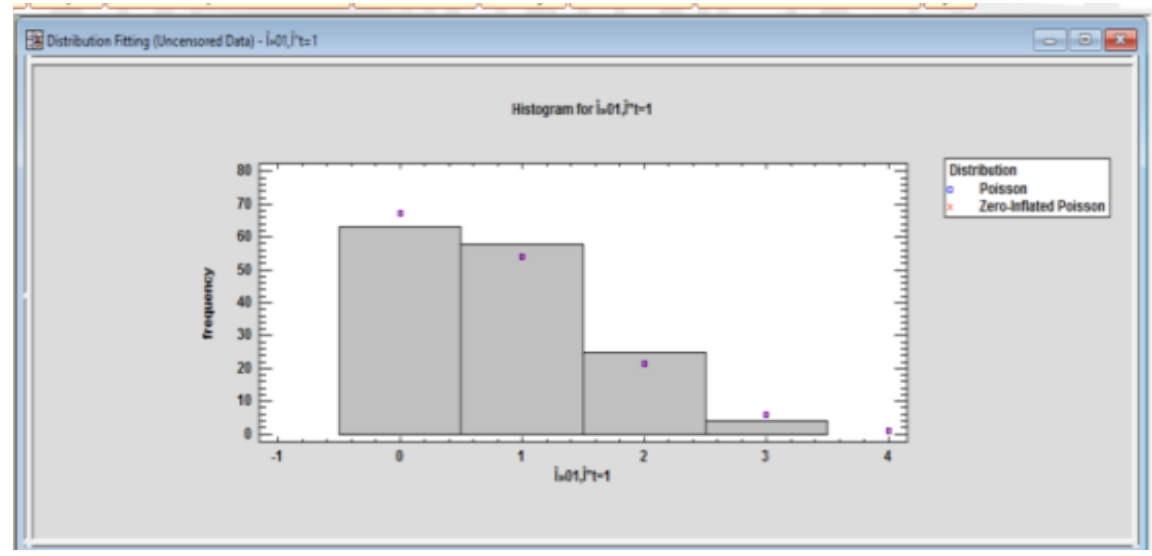

Figure 4: transition from 0 to 1

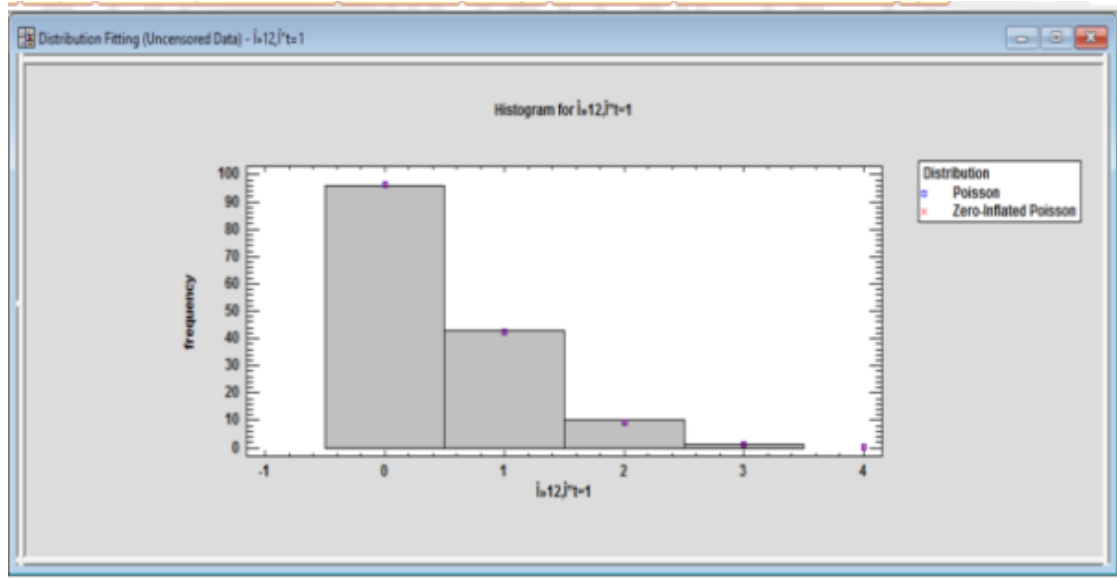

Figure 5: transition from 1 to 2

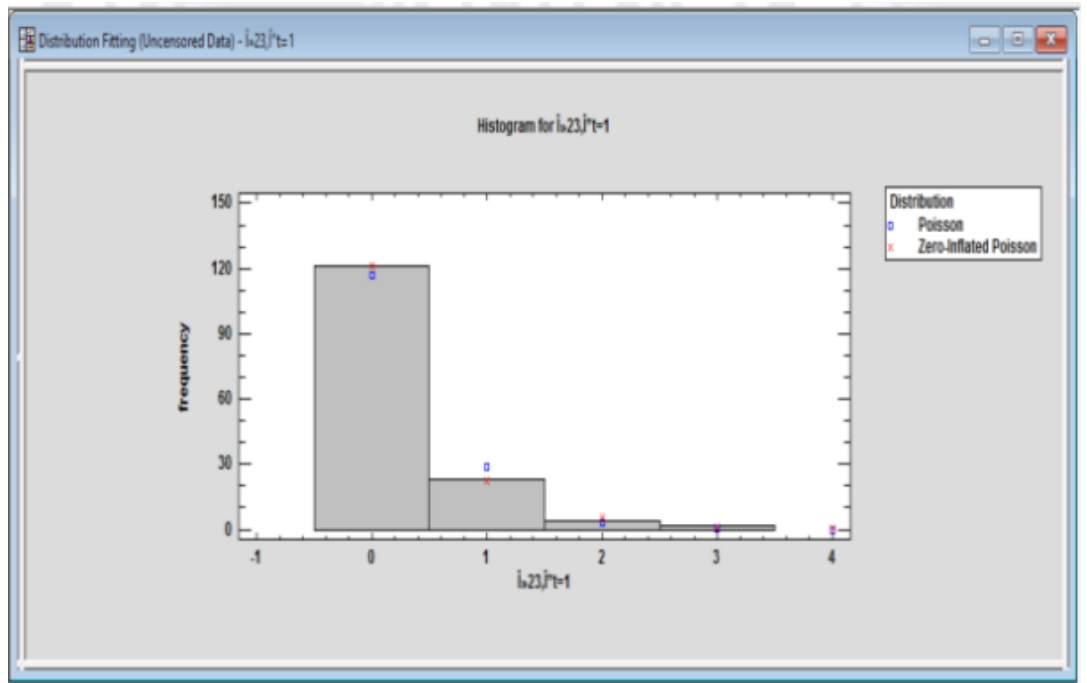

Figure 6: Transition from 2 to 3 


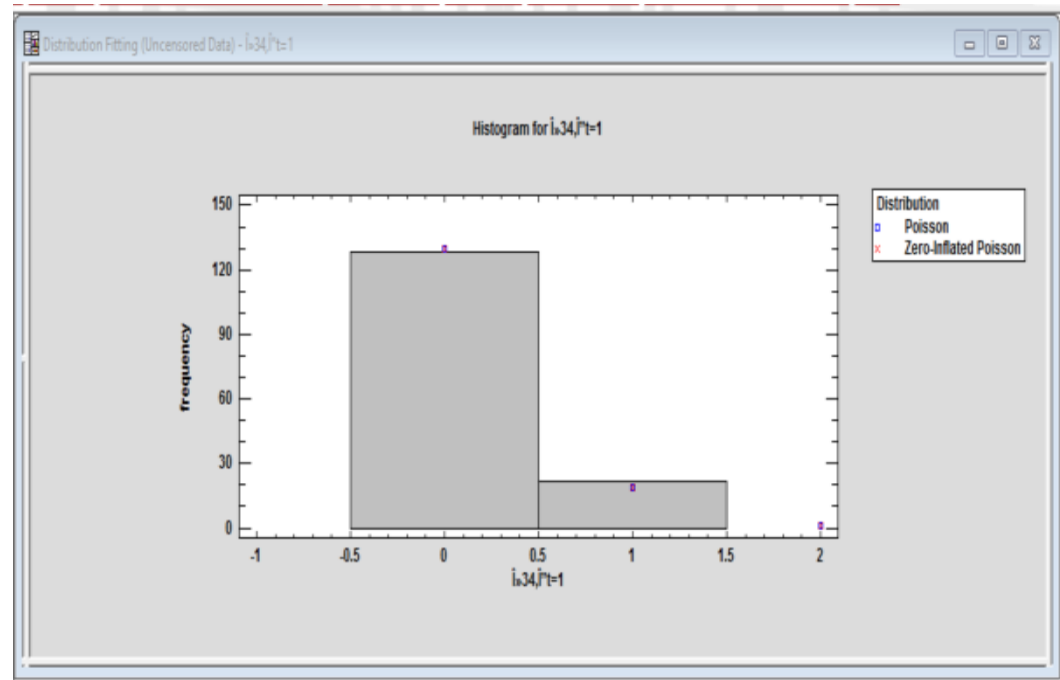

Figure 7: transition from 3 to 4

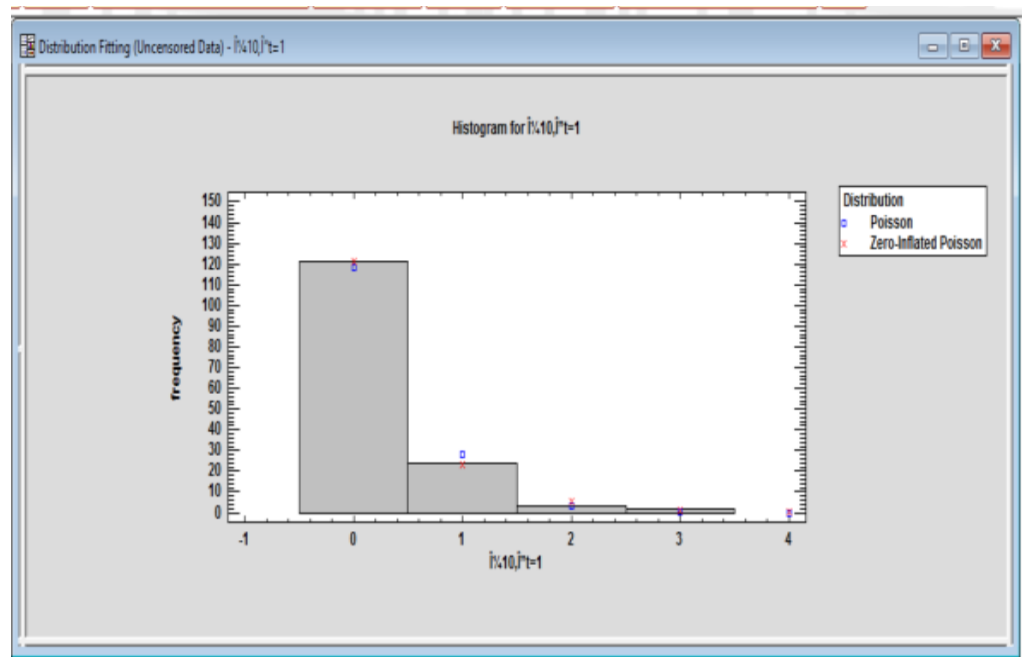

Figure 8: transition from 1 to 0

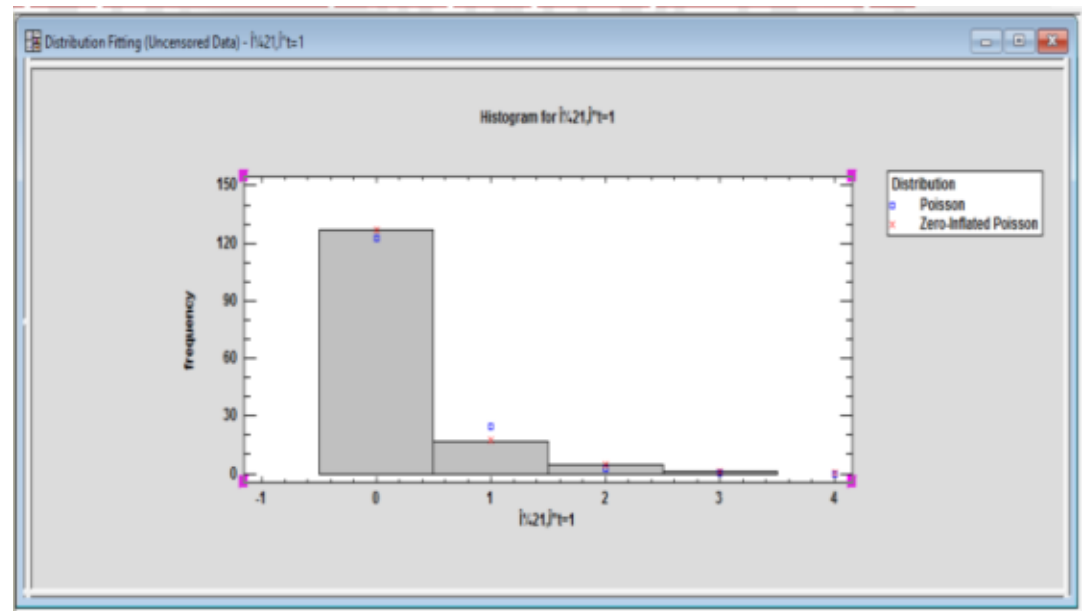

Figure 9: transition from 2 to 1 


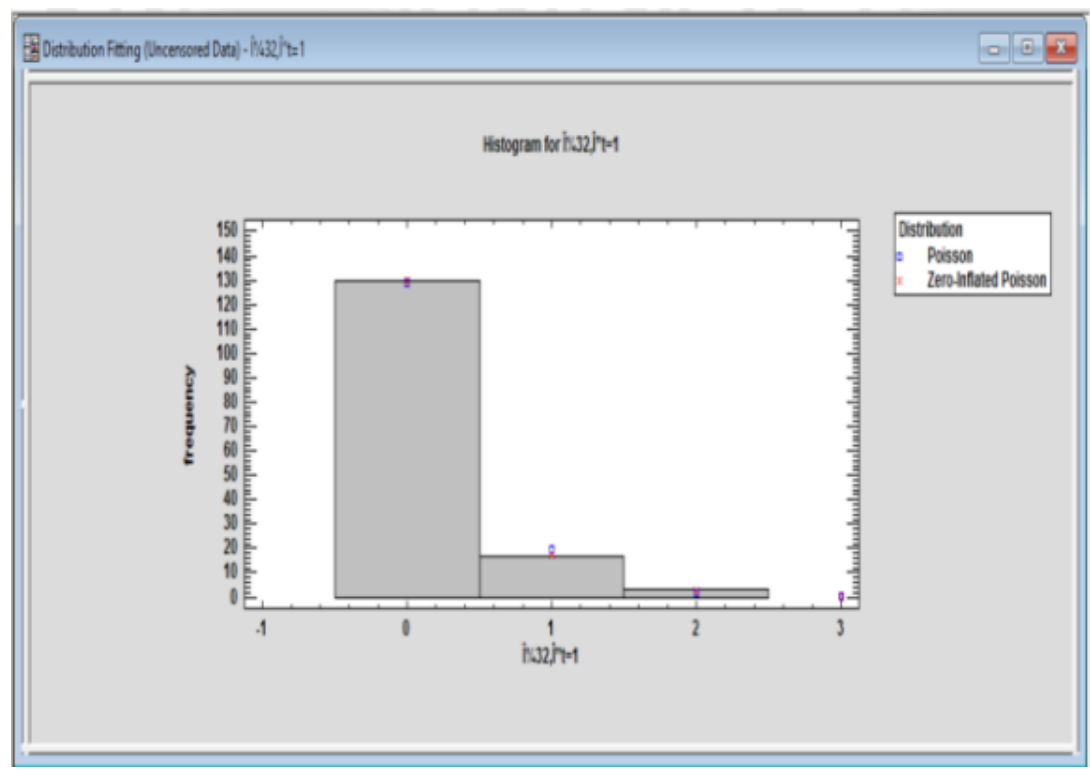

Figure 10: transition from 3 to 2

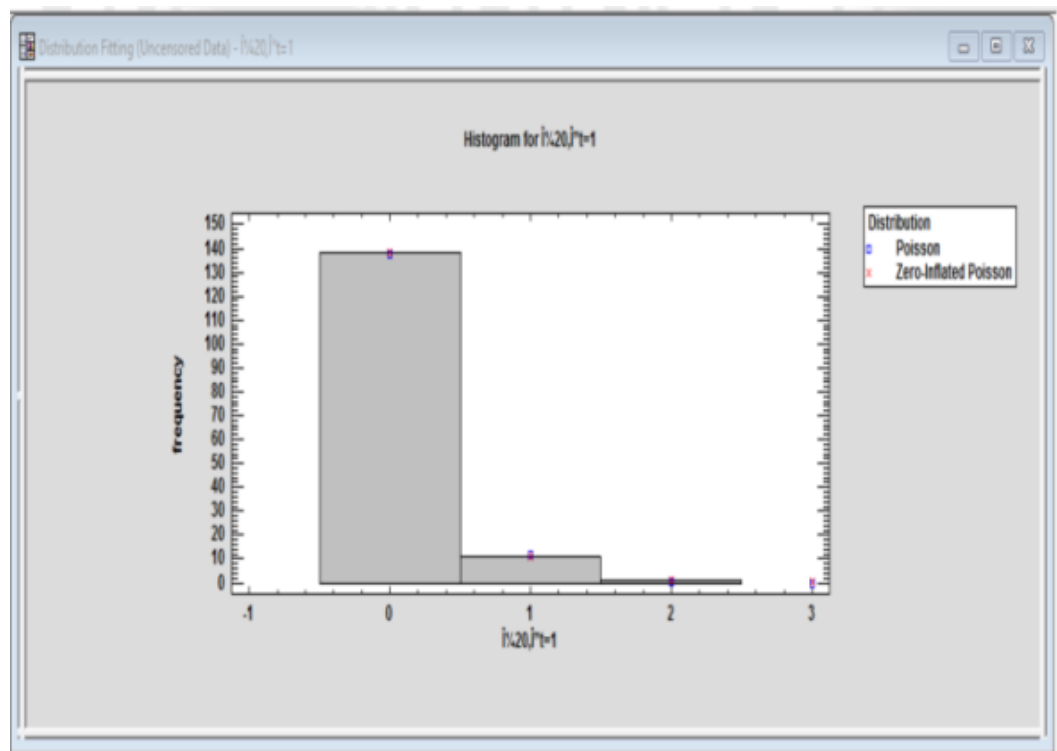

Figure 11: transition from 2 to 0 


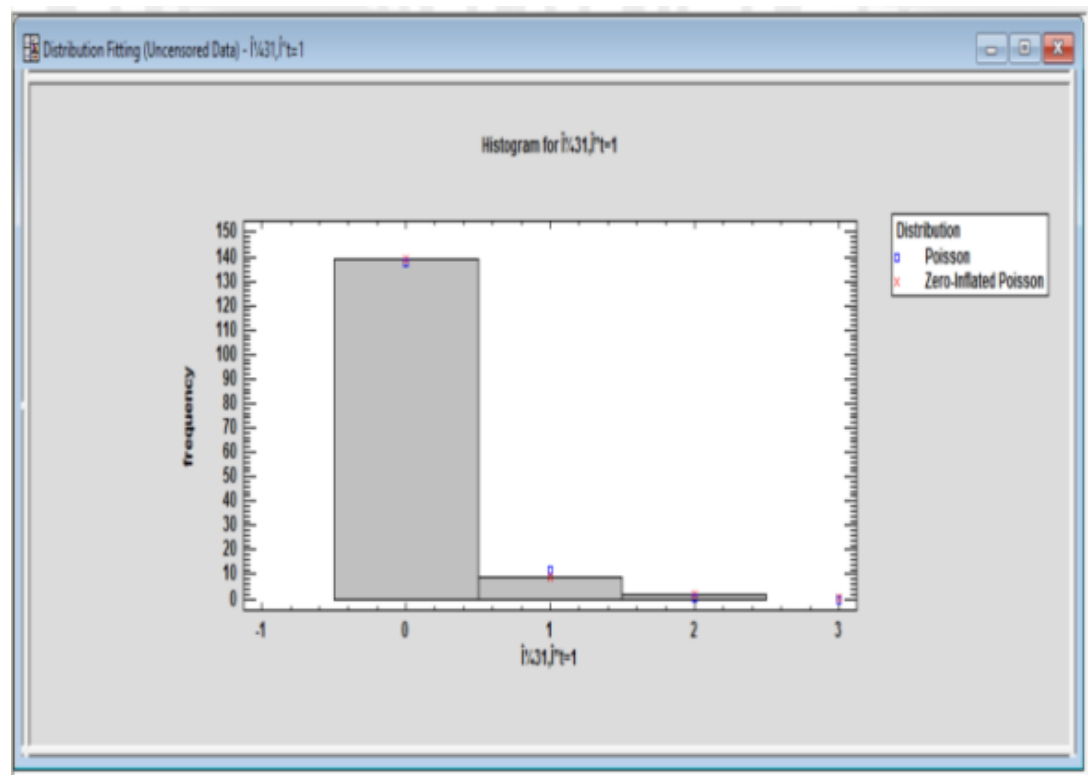

Figure 3:transition from 3 to 1

Lowess smoother illustrates that the relationships between each of the response rate and each variable is not strictly linear, but it is curvilinear relationship, with initial part of this relation being nearly horizontal and it starts to curve upwards at some predictor point located inside the second category of each predictor. The figures illustrating these relations are in supplementary materials from figure (13) to figure (21) for each response rate to the 7 variables. For example, relationship between number of transitions from state 0 to state 1 starts to bends up where each of the six predictors are located inside the second category; where age is approximately $\geq 37$, BMI is approximately $\geq 26$, LDL-chol is approximately $\geq 85 \mathrm{mg} / \mathrm{dL}$,
HOMA-IR is approximately $\geq 1.7$, systolic blood pressure is approximately $142 \mathrm{mmHg}$, and diastolic blood pressure is approximately $\geq 85 \mathrm{mmHg}$. All these values are located in the second category. This can give good orientation to the functional form of the variables to be used in the regression model and avoid the misspecification resulting from malfunctional form of the predictors. In this work the restricted cubic splines are used for the predictors with 5 knots using Harrell approach which is the default procedure utilized by Stata 14 software. The locations of knots are illustrated in table (10) and correlations between the transformed variables are presented in table (11).

Table (10): location of knots for specified variables using Harrell approach (the default used in Stata 14)

\begin{tabular}{|c|c|c|c|c|c|}
\hline & Knot 1 & Knot 2 & Knot 3 & Knot 4 & Knot 5 \\
\hline LDL-chol & 71.22 & 83.7 & 94.62 & 104.48 & 124.14 \\
\hline HOMA2-IR & 1.09 & 1.8 & 2.26 & 2.75 & 3.48 \\
\hline sysBloodPr. & 133.09 & 143.88 & 149.41 & 255.58 & 168.04 \\
\hline Dias b lood Pr. & 74.45 & 87.44 & 94.07 & 101.11 & 114.49 \\
\hline
\end{tabular}

Table (11): correlation between the transformed variables used in the Poisson regression models

\begin{tabular}{|c|c|c|c|c|c|}
\hline & LDLsp2 & HOMAsp1 & SYSsp2 & HOMAsp2 & \\
\hline LDLsp2 & 1 & & & \\
\hline HOMAsp1 & .8572 & 1 & & .9908 \\
\hline sysSP2 & .9959 & .8674 & 1 & 1 \\
\hline HOMAsp2 & .9893 & & .995 & 1 \\
\hline DiasSP2 & .9944 & & .9929 & 1 \\
\hline
\end{tabular}

The Poisson regression was applied using the observed counts of the transition counts matrix as response variable, and the following results are obtained as discussed below in the next section.

\section{Results and Discussion:}

In the next discussion, the results of running Poisson regression to obtain the following estimated counts are demonstrated. Running Poisson regression on these transformed variables gives the estimated counts shown in table (13): 
Table 13: the estimated counts for each transition

\begin{tabular}{|c|c|c|c|c|c|c|c|c|c|}
\hline Counts & $\begin{array}{c}\text { Transition } \\
\mathbf{0 \rightarrow \mathbf { 1 }}\end{array}$ & $\begin{array}{c}\text { Transition } \\
\mathbf{1 \rightarrow \mathbf { 2 }}\end{array}$ & $\begin{array}{c}\text { Transition } \\
\mathbf{2} \rightarrow \mathbf{3}\end{array}$ & $\begin{array}{c}\text { Transition } \\
\mathbf{3 \rightarrow \mathbf { 4 }}\end{array}$ & $\begin{array}{c}\text { Transition } \\
\mathbf{1 \rightarrow \mathbf { 0 }}\end{array}$ & $\begin{array}{c}\text { Transition } \\
\mathbf{2 \rightarrow \mathbf { 1 }}\end{array}$ & $\begin{array}{c}\text { Transition } \\
\mathbf{3 \rightarrow \mathbf { 2 }}\end{array}$ & $\begin{array}{c}\text { Transition } \\
\mathbf{2} \rightarrow \mathbf{0}\end{array}$ & $\begin{array}{c}\text { Transition } \\
\mathbf{3 \rightarrow \mathbf { 1 }}\end{array}$ \\
\hline $\mathbf{0}$ & 75 & 102 & 125 & 133 & 126 & 132 & 135 & 140 & 140 \\
\hline $\mathbf{1}$ & 34 & 35 & 18 & 14 & 15 & 12 & 12 & 8 & 7 \\
\hline $\mathbf{2}$ & 37 & 11 & 4 & 3 & 7 & 4 & 2 & 2 & 3 \\
\hline $\mathbf{3}$ & 4 & 1 & 3 & 0 & 1 & 2 & 1 & 0 & 0 \\
\hline $\mathbf{4}$ & 0 & 1 & 0 & 0 & 1 & 0 & 0 & 0 & 0 \\
\hline Total & 150 & 150 & 150 & 150 & 150 & 150 & 150 & 150 & 150 \\
\hline
\end{tabular}

The results for each transition are demonstrated in supplementary tables from table 14 up to table 22, and accompanied by discussion of these results to illustrate the importance of conducting such regression. [See supplementary materials for tables from table (14) to table (22) and associated discussion].

The comparison between the distribution of the response rates and the estimated rates is illustrated in table (23) (See supplementary materials).

As the estimated rates approximately equal the observed rates obtained by CTMC especially when using the initial rates calculated as $\theta_{0}=\frac{n_{i j r}}{n_{i+}}$ where the $n_{i j r}$ is the transition counts from state $i$ to state $j$ and the $n_{i+}$ is the total marginal transition counts out of this state $i$, as verified by the author; Iman Attia in previous 2 papers, and assuming that the marginal counts are the same, so the estimated $\mathrm{Q}$ transition rate matrix according to the estimated counts obtained by fitting Poisson regression is:

$Q=\left[\begin{array}{ccccc}-.059 & .059 & 0 & 0 & 0 \\ .029 & -.080 & .051 & 0 & 0 \\ .015 & .033 & -.093 & .045 & 0 \\ 0 & .108 & .158 & -.409 & .167 \\ 0 & 0 & 0 & 0 & 0\end{array}\right]$ where

$\lambda_{01}=\frac{120}{2050}=.059, \lambda_{12}=\frac{64}{1247}=.051, \lambda_{23}=\frac{35}{783}=.045$,

$\lambda_{34}=\frac{20}{120}=.167, \mu_{10}=\frac{36}{1247}=.029, \mu_{21}=\frac{26}{783}=.033$ $\mu_{32}=\frac{19}{120}=.158, \mu_{20}=\frac{12}{783}=.015, \mu_{31}=\frac{13}{120}=.108$

Probability transition matrix is obtained from exponentiating this Q matrix after 1 year:

$P(t=1)=\left[\begin{array}{ccccc}.9435 & .0551 & .0014 & 0 & 0 \\ .0274 & .9247 & .0469 & .0009 & .0001 \\ .0144 & .0327 & .9149 & .0348 & .0032 \\ .0023 & .0863 & .1245 & .6512 & .1357 \\ 0 & 0 & 0 & 0 & 1\end{array}\right]$

To calculate goodness of fit for multistate model used in this model, it is like the procedure used in contingency table, and it is calculated in each interval and then summed:

Step1: $H_{0}=$ future state does not depend on the current state $H_{1}=$ future state does depend on the current state.

Step2: Calculate the

$p_{i j}(\Delta t=1)=\left[\begin{array}{ccccc}.9435 & .0551 & .0014 & 0 & 0 \\ .0274 & .9247 & .0469 & .0009 & .0001 \\ .0144 & .0327 & .9149 & .0348 & .0032 \\ .0023 & .0863 & .1245 & .6512 & .1357 \\ 0 & 0 & 0 & 0 & 1\end{array}\right]$

Using exponentiation of the estimated Q matrix

Step3: Calculate the expected counts in this interval by multiplying each row in the probability matrix with the corresponding total marginal counts in the observed transition counts matrix in the same interval to get the expected counts as in the following table (24)

Table(24): expected counts of transition

\begin{tabular}{|l|l|l|l|l|l|l|}
\hline & State 0 & State 1 & State 2 & State 3 & State 4 & Total \\
\hline State 0 & 1934.175 & 112.955 & 2.87 & 0 & 0 & 2050 \\
\hline State 1 & 34.1678 & 1153.101 & 58.4843 & 1.1223 & 0.1247 & 1247 \\
\hline State 2 & 11.2752 & 25.6041 & 716.3667 & 27.2484 & 2.5056 & 783 \\
\hline State 3 & .276 & 10.356 & 14.94 & 78.144 & 16.284 & 120 \\
\hline State 4 & 0 & 0 & 0 & 0 & 0 & 0 \\
\hline
\end{tabular}

Step4: apply $\sum_{i=1}^{5} \sum_{j=1}^{5} \frac{\left(o_{i j}-E_{i j}\right)^{2}}{E_{i j}}=1140.097 \sim \chi_{(5-1)(5-1)(.05)}^{2}$

Therefore,from the above results the null hypothesis is rejected while the alternative hypothesis is accepted and the model fits the data that is to mean the future state depends on the current state with the estimated transition rates and probability matrices as obtained.
Of those patients starting at F0, only $5.51 \%$ will move to $\mathrm{F} 1$ in one year, this declines to $4.69 \%$ of patients starting at F1 moving to $\mathrm{F} 2$, while $3.48 \%$ of patients starting at $\mathrm{F} 2$ will move to $\mathrm{F} 3$; however, $13.57 \%$ of patients starting in F3 will move to $\mathrm{F} 4$, and this high percentage of patients moving towards advanced fibrosis may be due to the fact that advanced fibrosis is considered to be F3 and F4 and once the patient reaches F3, his chance to progress to F4 is higher than being in any starting stage considered less advanced fibrosis including F0 to F2 ( by 
definition ), and this is obvious as shown by incidence rate ratio of this transition being the highest $(5.237 \mathrm{e}+6)$. It is shown that progression from $\mathrm{F} 0$ to $\mathrm{F} 1$ and from $\mathrm{F} 1$ to $\mathrm{F} 2$ is approximately equal, while transition from F2 to F3 is less and this may be to more aggressive intervention taken by the patients to hinder the progression of fibrosis by applying more intensive lifestyle modifications, but once the patient reaches stage F3 the progression to F4 is by far the most among the forward transitions. There are $2.74 \%$ of patients starting at F1 will move to $\mathrm{F} 0$ while this percentage decreases to $1.44 \%$ if starting at $\mathrm{F} 2$, and it is even less if starting at F3 (only .23\% of patients can achieve this task); hence it is more feasible to move from F1 to $\mathrm{F} 0$ than to move from $\mathrm{F} 2$ to $\mathrm{F} 0$ than to move from $\mathrm{F} 3$ to F0; that is to mean, the more advanced the stage of fibrosis the patient experiences, the less likely movement to F0 he affords to do. There is a paradox if the starting stage is F2 or F3 to F1. The movement to $\mathrm{F} 1$ is more obvious if the patient is in F3 (8.63\% of patients move to F1) than if he is in F2 ( $3.27 \%$ of patients move to $\mathrm{F} 1)$; therefore, the more advanced fibrosis stage the patient recognizes, the more likely movement to F1 he can do, and may be this is due to the extensive lifestyle modification he performs to achieve less degree of fibrosis, but it remains a little bit difficult to reach F0 ( only .23\% of patient can move from F3 to F0). It is also noted that $2.74 \%$ of patients move from $\mathrm{F} 1$ to $\mathrm{F} 0,3.27 \%$ of patients move from $\mathrm{F} 2$ to $\mathrm{F} 1$ while $12.45 \%$ of patients move from F3 to F2 ; in other words the more advanced the fibrosis stage is, the more likely the movement to the immediately previous stage is. Moreover if the starting stage is $\mathrm{F} 3$, then $13.57 \%$ of these patients move to $\mathrm{F} 4$, a little bit higher than moving to $\mathrm{F} 2$ (12.45\% of the patients); whereas, movement to F1 and F0 declines $(8.63 \%$ of the patients and $.23 \%$ of the patients respectively, approximately movement to $\mathrm{F} 0$ is $2.66 \%$ that to $\mathrm{F} 1$ ). Of those patients starting in $\mathrm{F} 2,3.48 \%$ move to $\mathrm{F} 3$, a little bit more than moving to $\mathrm{F} 1$ (3.27\% of patients); nevertheless, movement to F0 is almost $44 \%$ that to $\mathrm{F} 1$ ( $1.44 \%$ of the patients move to $\mathrm{F} 0$ ).

Mean time spent by the patient in state 0 is approximately 17 years that declines to 12 years and 6 months spent in state 1 , which further declines to approximately 10 years and 9 months spent in state 2 , and ultimately reaching 2 years and 3.7 months spent in state 3 . It is shown that, there is decrease in time spent in each stage as the disease process evolves over time. This huge rapid decline in time spent in state 3 is due to advanced fibrosis induced by dead hepatocytes, especially if no treatment is introduced like: lifestyle modification ,risk factors treatment, as well as anti-inflammatory and anti-fibrotic drugs, and if so, it is a matter of time to reach state 4 , which is irreversible stage of damaged liver cells that will soon manifest with reduction in liver cell functions, and may be to hepatocellular carcinoma, and eventually death, if not managed with liver transplantation.

\section{Conclusions:}

Insulin resistance is a key stone for triggering all these abnormalities, the more sensitive the body cells is to insulin, the less likely the complications of NALFD will develop. The effect of risk factors or covariates as a mainstay players, like: increased insulin resistance, hyperlipidemia with increased LDL-cholesterol, high systolic and diastolic blood pressure are thoroughly explained using the Poisson regression model combined with CTMC. As concluded from the hypothetical model that for every unit increase in the transformed HOMA, the incidence rate ratio for transition from state 0 to state 1 is increased by $5909.7 \%$ and this elevation is kept rising while moving forward from subsequent state to the immediately next state, that is to mean, for every unit increase in the transformed HOMA, the incidence rate ratio (IRR) for transition from state 1 to state 2 is increased by $24017.9 \%$, while for the transition from state 2 to state 3 , it is increased by $47931.8 \%$, and for transition from state 3 to state 4 it is increased by $5237498.4 \%$. This increment is almost always highly statistically significant. This is in comparison with transformed LDL, as for every unit increase in the transformed LDL, the IRR for transition from state 0 to state 1 is increased by $68.7 \%$, while for the transition from state 1 to state 2 , it is increased by $36.4 \%$, and for transition from state 3 to state 4 it is increased by $57.1 \%$. And it is only highly statistically significant for transition from state 3 to state 4 . However the systolic blood pressure is almost highly statistically significant for the transition from state 2 to state 3 as obvious by for every unit increase in the transformed systolic pressure, the IRR for this transition to occur is increased by $1114.3 \%$. Moreover, for every unit decrease in the transformed HOMA, the IRR for transition from state 1 to state 0 is increased by $1.1 \%$, for transition from state 2 to state 1 it is increased by $3.7 \%$, for transition from state 3 to state 2 it is increased by $0.5 \%$, for transition from state 2 to state 0 it is increased by $6.6 \%$, and for transition from state 3 to state 1 it is increased by $8.4 \%$. This emphasizes that better control of insulin resistance helps the patient to reverse his condition. To sum up, the precipitating factors should be rigorously and extensively treated and controlled by life style modifications represented by dietary restriction of high calorie diet and sedentary life, thus the predisposed persons should consume healthy diets and regularly practicing physical exercises suitable for their medical conditions. The newly discovered drugs like anti-fibrotic drugs that treat the fibrotic changes in the liver are promising drugs and await further longitudinal studies, to reveal the most effective protocol, by which they are administered to the patients, for better control of the rate of progression of liver fibrosis. This control keeps the patient out of loss of liver functions, and subsequently away from end stage liver disease, which necessitates liver transplantation with all its accompanying post transplantation complications.

\section{Hint (programs and supplementary materials):}

The above example is published with Stata data, accompanied do file, as well as the supplementary materials file on the code ocean sit with the following URL : Codeocean.com/capsule/4752445/tree/v1 


\section{Abbreviations:}

CC: compensated cirrhosis (stage 4), CTMC: continuous time Markov chains, DCC: decompensated cirrhosis (stage 5), EM: extramortality (stage9), HCC: hepato-cellular carcinoma (stage 8), LT: liver transplant (stage 6), NAFLD: nonalcoholic fatty liver disease, NAFLD-NO FB: nonalcoholic fatty liver disease with no fibrosis (stage1), NASH: nonalcoholic steatohepatitis, NASH-NO FB: nonalcoholic steatohepatitis with no fibrosis (stage2), NASH-FB: nonalcoholic steatohepatitis with fibrosis (stage3), PLT : post liver transplant (stage7), T2DM: type 2 diabetes mellitus.

\section{Declarations:}

\section{Ethics approval and consent to participate}

Not applicable.

\section{Consent for publication}

Not applicable

\section{Availability of data and material}

Not applicable. Data sharing were not applicable to this article as no datasets were generated or analyzed during the current study.

\section{Competing interests}

The author declares that I have no competing interests.

\section{Funding}

No funding resource. No funding roles in the design of the study and collection, analysis, and interpretation of data and in writing the manuscript are declared

\section{Authors' contribution}

I am the author who has carried the mathematical analysis as well as applying these mathematical statistical concepts on the hypothetical example.

\section{Acknowledgement}

Not applicable

\section{References}

[1] Younossi, Zobair M., Deirdre Blissett, Robert Blissett, Linda Henry, Maria Stepanova, Youssef Younossi, Andrei Racila, Sharon Hunt, and Rachel Beckerman. 2016. "The Economic and Clinical Burden of Nonalcoholic Fatty Liver Disease in the United States and Europe." Hepatology 64(5):1577-86.

[2] Younossi, Zobair M., Radhika P. Tampi, Andrei Racila, Ying Qiu, Leah Burns, Issah Younossi, and Fatema Nader. 2020. "Economic and Clinical Burden of Nonalcoholic Steatohepatitis in Patients with Type 2 Diabetes in the US." Diabetes Care 43(2):283-89.

[3] Singh, Siddharth, Alina M. Allen, Zhen Wang, Larry J. Prokop, Mohammad H. Murad, and Rohit Loomba. 2015. "Fibrosis Progression in Nonalcoholic Fatty Liver vs Nonalcoholic Steatohepatitis: A Systematic Review and Meta-Analysis of Paired-Biopsy Studies." Clinical Gastroenterology and Hepatology 13(4):643-54.

[4] Kalbfleisch, J. D., and Jerald Franklin Lawless. 1985. "The Analysis of Panel Data under a Markov Assumption." Journal of the American Statistical Association 80(392):863-71.

[5] Attia, I.M. 2021. "New Method Utilizing Continuous Time Markov Chains to Analyze Evolution in the Nine States
Model of Non-Alcoholic Fatty Liver Diease." International Journal of Research in Engineering And Sicience. vol:9,issue(9):56-106. Associated published MATLAB code in the code ocean site with the following URL: codeocean.com/capsule/7628018/tree/v1

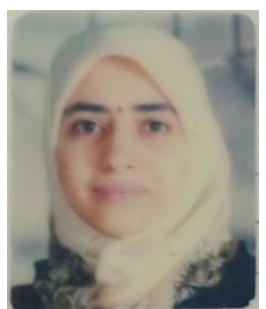

Iman Attia is currently M.Sc student in Faculty of Graduate Studies of Statistical Research, Mathematical Statistics Department, Cairo University. In 2001, she received M.Sc. degree in internal medicine, Cairo University. In 2015, she received Bachelor's degree in Computer Science and Information Technology from the Egyptian Electronic-learning University (EELU). In 2017, she received Diploma in statistics from Institute of Statistical studies and Research, Cairo University. Her research interests include biostatistics, bioinformatics and machine learning. 